\title{
Philosophiques
}

\section{Giroux, Dalie, Lemieux, René, et Chénier, Pierre-Luc (dir.). Contr'hommage pour Gilles Deleuze - Nouvelles lectures, nouvelles écritures, Québec, PUL, 2009}

\section{Jean-Claude Simard}

Volume 38, numéro 1, printemps 2011

URI : https://id.erudit.org/iderudit/1005738ar

DOI : https://doi.org/10.7202/1005738ar

Aller au sommaire du numéro

Éditeur(s)

Société de philosophie du Québec

ISSN

0316-2923 (imprimé)

1492-1391 (numérique)

Découvrir la revue

Citer ce compte rendu

Simard, J.-C. (2011). Compte rendu de [Giroux, Dalie, Lemieux, René, et Chénier, Pierre-Luc (dir.). Contr'hommage pour Gilles Deleuze - Nouvelles lectures,

nouvelles écritures, Québec, PUL, 2009]. Philosophiques, 38(1), 357-365.

https://doi.org/10.7202/1005738ar

Ce document est protégé par la loi sur le droit d'auteur. L'utilisation des services d'Érudit (y compris la reproduction) est assujettie à sa politique d'utilisation que vous pouvez consulter en ligne.

https://apropos.erudit.org/fr/usagers/politique-dutilisation/ 
Hegel, c'est l'existence de l'historialité qui apparaît problématique, car il n'est pas rendu compte de la distance qui sépare les penseurs, et cela apparaît précisément dans sa confrontation avec Heidegger. Comme le montre l'auteure, Hegel pense le dialogue sur le mode de la communauté politique, mais cela ne rend pas compte du temps qui sépare les protagonistes du dialogue transhistorique qu'est l'histoire de la philosophie. En envisageant le dialogue non pas comme lieu politique, mais comme site de la pensée, Heidegger serait plus à même de rendre compte de l'évènement (Ereignis) de la pensée. À la suite de Hölderlin mettant en avant la destination (Geschick) de la pensée, il s'attacherait à creuser l'écart en situant le lieu d'où l'autre fait signe. Comme l'écrit l'auteure, "la situation de l'autre permet [...] l'éclaircissement du site propre, et l'explication mesure l'écart historique des deux » (p. 196).

En définitive, si les deux penseurs partagent tout deux un même souci d'historialité, leur conflit permet de les problématiser et de les transformer au fur et à mesure de la confrontation. L'entre-deux dans lequel se positionne l'auteure est ainsi celui d'un dialogue dans lequel elle pense avec des "amis", des penseurs dont elle reconnaît le caractère vivant, "mais en ennemi plutôt qu'en partisan» (p. 204). Le conflit apparaît ainsi au cœur même de l'historialité, et plus généralement du dialogue philosophique, dont il constituerait le moteur. "Heidegger contre Hegel. Les Irréconciliables» constitue une illustration remarquable de cette thèse tant il ouvre des pistes de pensée intéressantes aussi bien pour les chercheurs heideggériens que pour les hégéliens, et plus généralement pour quiconque se veut ami de la sagesse.

GUILLAUME LEJEUNE

Université libre de Bruxelles

Giroux, Dalie, Lemieux, René, et Chénier, Pierre-Luc (dir.). Contr'hommage pour Gilles Deleuze - Nouvelles lectures, nouvelles écritures, Québec, PUL, 2009.

Jamais auparavant un recueil consacré à Deleuze n'avait paru au Québec. L'initiative était donc louable, mais le résultat est malheureusement mitigé. Issu d'un colloque tenu à Trois-Rivières en 2007, ce collectif regroupe en effet douze contributions de valeur très inégale. Divisé en trois sections, le recueil veut ouvrir de nouvelles perspectives sur cette œuvre à la fois vaste, multiforme et d'une technicité élevée. Cependant, il souffre de deux handicaps. Le premier, c'est qu'on y trouve à la fois des chercheurs confirmés et des étudiants de deuxième et troisième cycles, de sorte que les niveaux se croisent sans toujours s'accorder. Le second, c'est que, afin d'ouvrir ces horizons inédits, seules quatre personnes travaillant en philosophie ont été conviées à participer. Les autres collaborations proviennent de domaines 
variés: politique, études cinématographiques, arts visuels et création littéraire. Or l'œuvre de Deleuze propose un vocabulaire riche, elle développe des argumentations serrées et crée des concepts complexes, bref, elle constitue un massif d'un abord malaisé. Aussi, malgré un caractère parfois original, certaines contributions peinent-elles à s'élever à la hauteur de leur sujet. Ces deux limites sont parfaitement illustrées par le fait qu'aucune des têtes dirigeantes de l'ouvrage ne travaille en philosophie; toutes trois sont issues du domaine politique, et une seule est enseignante (Université d'Ottawa), les deux autres étant des doctorants. Conscients du second écueil, les responsables de la publication ont voulu tabler sur la fécondité de la pensée deleuzienne. Aussi les deuxième et troisième sections du recueil cherchentelles à montrer l'usage que l'on en peut faire dans divers domaines. Bref, au lieu de remonter à la source pour proposer de nouvelles interprétations de l'œuvre de Deleuze ou des angles originaux, comme dans la première partie, ces praticiens venus d'horizons divers ont plutôt privilégié ses prolongements en aval, ce qui est bien illustré par le choix du titre, le terme "contr'hommage» constituant, de la part de ces familiers de Deleuze, un clin d'œil volontaire au Contr'un de La Boétie, ami indéfectible de Montaigne. On a voulu, dit la préface, "mettre à l'œuvre Deleuze» (p. 14; italique dans le texte). Bref, nous sommes ici en présence d'un objet culturel intéressant et, en un sens, inédit, mais hybride à plus d'un titre.

Cela dit, ce pari présente tout de même des mérites certains et, parmi les textes notables du recueil, il faut tout d'abord souligner l'analyse solide d'Alain Beaulieu ( «La pratique deleuzienne de l'histoire», p. 3-21), spécialiste reconnu de Deleuze, qui ouvre la première partie de l'ouvrage: «Lectures». On sait que la philosophie entretient un rapport privilégié à sa propre histoire. Or, en ce domaine, l'approche de Deleuze est restée unique dans le monde européen. Il se penchait en effet, avec une rare pénétration, sur les systèmes philosophiques du passé, tout en en déplaçant subtilement l'architectonique, afin de montrer leur parfaite actualité. Après avoir brièvement mis en lumière cette pratique très originale, Beaulieu aborde la conception deleuzienne de l'éducation, un domaine connexe encore en friche, et il montre comment elle refuse toute normalisation de la discipline, parce que Deleuze croit à une philosophie expérimentale, hors institution. La jonction entre histoire et éducation se réalise grâce à la "perspective microscopique » (p. 11-12), un rejet des usages macroscopiques de l'histoire de la philosophie, comme ceux de Heidegger, Derrida, Adorno ou Husserl. L'avenue empruntée par Deleuze mène à une philosophie transhistorique, inséparable d'une dramaturgie de l'histoire, par quoi il faut entendre une partition du champ historique entre héros de la pensée authentique (par exemple Nietzsche, Hume, Spinoza, Foucault), opposants (Hegel, Freud, Wittgenstein) et représentants d'une sorte d'affrontement amical (tout le courant phénoménologique). Bref, les différents penseurs de l'histoire de la philosophie assument, de ce point de vue, une fonction héroïque, antagonique ou 
ludique (p. 16). C'est ce qui les rend intempestifs, comme voulait d'ailleurs le devenir la philosophie même de Deleuze.

Les deux études suivantes de la première partie se penchent sur des objets nettement plus circonscrits. Sjoerd Van Tuinen ( Pris dans une sorte de serpentin. Le concept deleuzien de maniérisme entre Bacon et le baroque ») analyse la notion artistique de maniérisme à partir de deux ouvrages de Deleuze (Francis Bacon - Logique de la sensation, $1981^{1}$ et Le pli Leibniz et le baroque, 1988), tandis que, pour sa part, Erik Bordeleau (« La Chine et la ligne. Une étude de la référence chinoise dans Mille plateaux») interroge la littérature et l'art chinois (entre autres les œuvres de Lu Xun) pour évaluer l'usage que, dans leur ouvrage à prétention historique, Deleuze et Guattari font de la référence à l'Empire du Milieu. En revanche, la quatrième et dernière étude de cette section, celle d'Andeas Krebs ( «e Corps sans Organes. Mobilisation pour une méthodologie d'observation sociale », p. 63-82), s'attaque à un concept fondamental chez Deleuze, réputé quoiqu'un brin mystérieux, le Corps sans Organes (symbolisé par CsO dans Mille plateaux). Avant d'aborder son étude de cette notion, qui a fait couler beaucoup d'encre, un petit rappel s'impose.

Emprunté à Artaud, le $\mathrm{CsO}$ figure en bonne place dans de nombreux ouvrages de Deleuze, écrits seul ou en collaboration avec Guattari. Plus profond que le corps vécu de la phénoménologie, c'est un «au-delà de l'organisme", une "limite du corps vécu» ${ }^{2}$. C'est encore un "organisme sans parties ", un corps intensif, sans surface, où se confondent intérieur et extérieur, contenant et contenu'. Pour Deleuze et Guattari, il s'agit d'un acquis essentiel de la schizo-analyse et, dans L'anti-CEdipe, ils le mettent en relation avec le concept central de machine désirante. Le CsO se généralise ensuite, passant de l'expérience du corps schizophrénique - celle, par exemple, d'Artaud - au désir à l'état brut, précédant toute captation. Pour préciser davantage, disons enfin que, bien qu'il s'agisse d'une expérience-limite impersonnelle, le $\mathrm{CsO}$ n'est pas une forme de transcendance, mais un idéal d'immanence totale, une corporalité, si l'on peut dire, à l'état pur".

1. Notons au passage que le système de renvois utilisé par Van Tuinen risque d'être un peu déroutant pour un non-initié. Ainsi, ou bien il utilise des reprises par une autre maison, sans mention de la publication initiale (le Francis Bacon de 1981, 2 vol., aux Éditions de la Différence, est remplacé par l'édition ultérieure de 2002, au Seuil), ou bien il fait appel à l'une ou l'autre des rééditions d'un même éditeur, mais sans l'indiquer (ainsi le Différence et répétition de 1968, aux PUF, est cité en bibliographie comme un ouvrage de 1997, tandis que Logique $d u$ sens est plutôt cité dans l'édition princeps de 1969). Ce flottement chronologique rend plusieurs passages de son texte difficilement compréhensibles, par exemple à la p. 32, n. 6, quand un commentateur analyse un ouvrage de Deleuze avant sa (supposée) parution...

2. Deleuze, Francis Bacon, op. cit., p. 33.

3. Deleuze, Logique du sens, Minuit, 1969, p. 106-108.

4. À ce propos, voir encore le chap. VI de Mille Plateaux, «Comment se faire un Corps sans Organes» (p. 185-204). 
Cela dit, quel est, dans ce texte, l'objectif de Krebs? Dans la foulée de plusieurs analystes anglo-saxons, qui ont assimilé divers éléments de la French Theory (Bennett, Holland, Massumi, etc.), il veut mettre au travail la pratique du $\mathrm{CsO}$ dans le domaine social. Pour ce faire, il analyse d'abord la façon dont, à partir du $\mathrm{CsO}$ et des flux vitaux, se constitue progressivement le sujet individuel. Pour Krebs, notons-le, le CsO est «la couche sur laquelle le désir s'enregistre» (p. 65). Après avoir ainsi rappelé la théorie de la constitution du sujet selon Deleuze et Guattari, il se demande pour quelle raison on voudrait se façonner un $\mathrm{CsO}$. Sa réponse est simple: ce nouveau $\mathrm{CsO}$ n'est pas l'originel, mais une construction qui permettra de libérer le flux des désirs et d'échapper à certains codages sociaux pernicieux, par exemple les préjugés envers les autochtones, les immigrants, les prestataires d'aide sociale, etc. En d'autres termes, la constitution du $\mathrm{CsO}$ "permet une dissolution du moi» (p. 71), étape cruciale dans une "éthique thérapeutique» (p. 73), laquelle constitue elle-même un palier vers "une théorie du changement social», issue des travaux de Deleuze et Guattari (p. 74). Cela acquis, Krebs propose ensuite le $\mathrm{CsO}$ comme technique d'observation empirique et d'intervention sociale, technique destinée à remplacer l'objectivité traditionnelle du chercheur, ou encore la séparation habituelle entre chercheur et objet de recherche. C'est là la partie la moins convaincante du texte, il faut bien le dire, et, dans les exemples offerts par l'auteur, on ne voit pas toujours très bien l'avantage qu'amènerait, pour la pratique sociale, la construction d'un $\mathrm{CsO}$ (à supposer, évidemment, que la chose soit possible, ce qui ne va pas du tout de soi). D'autant plus d'ailleurs que, pour exposer ensuite le résultat d'éventuelles recherches ainsi réalisées, à la place du traditionnel compte rendu scientifique, Krebs propose un style d'écriture qui, à la manière de Deleuze et Guattari, évacuerait la narration linéaire et le sujet fixe, détruisant par la même occasion "la distinction entre sujet et objet » (p. 81). Si ce type d'écriture éclatée, discontinue et rejetant toute perspective unique ou centrée convient parfaitement à des machines philosophiques subversives, comme L'anti-CEdipe ou encore Mille plateaux, son utilisation pour de futurs rapports de recherche risque d'engendrer un certain scepticisme...

Avec le texte de Krebs se termine la première partie de l'ouvrage. La deuxième, "Écritures", propose un registre entièrement différent. Il s'agit en effet d'expérimenter, à partir de différents domaines, de nouvelles possibilités, ou, dans la terminologie deleuzienne, de proposer des lignes de fuite, des formes de déterritorialisation. Il se compose de quatre écrits très dissemblables, parmi lesquels on a l'agréable surprise de trouver un texte du très controversé écrivain de science-fiction cyberpunk, Maurice G. Dantec, d'origine française, mais résidant à Montréal, catholique confirmés et, selon

5. Comme il le rappelle dans son texte, Dantec s'est fait baptiser en 2004, à l'âge de 45 ans, «selon le rite apostolique et romain» (p. 151). 
plusieurs commentateurs, réactionnaire impénitent ${ }^{6}$. À tout seigneur tout honneur, parlons d'abord de ce texte, "De la machine de $3^{\mathrm{e}}$ espèce aux humains du $4^{\mathrm{e}}$ type » (p. 147-158), qui clôt la deuxième partie de l'ouvrage. En tant que "praticien de la littérature", Dantec ne souhaite pas "écrire quelque exégèse que ce soit concernant Gilles Deleuze et son travail» (p. 147) ${ }^{7}$. Ce qui l'intéresse plutôt, c'est de témoigner de sa rencontre avec ce "grand philosophe de la singularité au $\mathrm{xx}^{\mathrm{e}}$ siècle» (p. 148; italique de Dantec), découvert par hasard dès l'adolescence, et dont le fructueux apport fut réactivé lors d'une autre rencontre, celle de Richard Pinhas. Ce Pinhas, un temps professeur de philosophie à la Sorbonne, est considéré comme un pionnier du rock électronique français. Le titre de certains de ses albums révèle d'ailleurs sa familiarité avec l'œuvre de Deleuze, un ami dont il fut d'abord l'élève: Rhizosphere (1979), De l'Un et du Multiple (2002), etc. De 1999 à 2003, lui et Dantec, musicien dans une vie antérieure, ont collaboré au projet Schizotrope, au nom évocateur, dont sont issus trois albums d'hommage à Deleuze, Dantec lisant des textes du philosophe sur un fond musical assuré par le groupe. L'écrivain signale d'ailleurs que, dès 1971, Deleuze lui-même avait lu un texte de Nietzsche sur l'un des albums de Pinhas. Dépassant le « simple travail de citation référentielle », c'est ce métissage des genres et ces recherches croisées que Dantec appelle une «machine de $3^{\text {e }}$ espèce» (p. 152, 151), alors que chacun, ventriloque, répercute le matériau actif d'un interlocuteur. Dans la dernière partie du texte, Dantec invoque le surhomme de Nietzsche, puis la reprise d'une philosophie de l'événement chez Deleuze, et il appelle de ses vœux la venue du post-humain, qu'il baptise d'un nom fleurant la science-fiction: l'homme du $4^{\mathrm{e}}$ type. Pour les directeurs de la publication, cet apport de Dantec, à la fois détonant et original, « résume à lui seul la problématique du contr'hommage » (p. 17-18), dont il traduit parfaitement l'esprit.

Dans la même veine, mais sous un mode différent, le texte expérimental de Claudine Vachon, «J'avance» (p. 111-125), explore librement les thématiques deleuziennes du désir et de l'errance. La facture de cette contribution n'est d'ailleurs pas sans rappeler Glas, de Derrida, car Vachon y juxtapose, sur deux pages en regard, poésie et récit, lesquels s'entrecroisent et entrent en résonance constante.

Pour sa part, Serge Cardinal, professeur au Département d'histoire de l'art et d'études cinématographiques de l'Université de Montréal, revient sur les deux tomes de la magistrale étude de Deleuze sur le cinéma, Cinéma I - L'image-mouvement (1983), et Cinéma II - L'image-temps (1985).

6. Une liste de ses lectures, mentionnée en p. 148, en donne d'ailleurs un indice assez probant: Bloy, Bernanos, Joseph de Maistre, etc.

7. Il ne pourra cependant résister à la tentation et, dans la dernière section de son essai, p. 153-158, il amorcera, lui aussi, une interprétation personnelle de certains concepts névralgiques de Deleuze. 
Dans un texte dense et suggestif, "Donner de l'image contre de l'argent, donner du temps contre des images » $(\mathrm{p} .127-145)^{8}$, il cherche à cerner, chez Deleuze, l'apport du cinéma à la philosophie. Dans un premier temps, il note que la pensée du philosophe repose sur deux grands principes: l'univocité de l'Être et l'universalité du devenir'. Selon lui, comme cette mise en mouvement de la pensée "tend à une ontologie "cinématographique" " (p. 128; c'est Cardinal qui souligne), il est assez normal que l'esthétique de Deleuze constitue une application étroite de sa philosophie générale. Et puisqu'il faut affirmer la vie, lors même que nous vivons dans un régime économique qui tend à la nier, il s'agit d'exténuer de l'intérieur le capitalisme. Appliqué au cinéma, cela signifie le pousser à la limite ou, en d'autres termes, donner de l'image contre de l'argent. Dans le volume 2 de son opus magnum sur le cinéma, Deleuze considère que le régime cinématographique moderne est déterminé, non plus par le mouvement, comme à son origine, mais par le temps. Et le concept qui, dès l'après-guerre, articule selon lui ce régime inédit, c'est l'image-cristal, diffraction du temps qui peut être parfaite (Ophuls), volontairement fêlée (Renoir), en gestation (Fellini) ou en décomposition (Visconti). Or, pour Cardinal, «c'est une nouvelle subjectivité nomade qui s'expérimente dans le cristal», celle "qui fait voir la démence du capital et en tire des possibilités de vie» (p. 134), celle aussi qui permet de donner du temps contre des images. C'est cette esthétique cinématographique, conçue comme "expérimentation éthique»(p. 138 sq.), qui intéresse Cardinal, et dont il cherche à tirer les conséquences.

Le dernier texte de cette deuxième partie, le plus long du recueil, s'intitule «Création et peuple: topologie du peuple manquant» (p. 85-110), et il est signé Pierre-Luc Chénier. En prenant comme point de départ un passage de L'image-temps sur le cinéma politique (p. 281-291), où est d'ailleurs évoqué le cas du Québec à travers l'œuvre de Perrault, l'auteur cherche à déterminer la façon dont, selon Deleuze, le langage et le cinéma peuvent favoriser l'affirmation d'un peuple minoritaire.

«Lecture ", la troisième et dernière section de l'ouvrage, change à nouveau de registre. Composée de trois textes, elle examine la façon dont Deleuze lit un auteur comme Kafka (Dalie Giroux), de quelle façon on l'a reçu aux États-Unis (Sylvano Santini), ou enfin la façon de lire Deleuze luimême (René Lemieux). Giroux (" "Tentation d'évasion hors de la sphère paternelle". Lecture et anarchie chez Gilles Deleuze », p. 177-190) analyse, dans son texte, les conditions facilitant la lecture politique d'une œuvre. Afin d'asseoir sa démonstration, elle prend à témoin le Kafka. Pour une littérature mineure (1975), de Deleuze et Guattari. On sait que l'interprète le plus connu de Kafka est Max Brod, écrivain et journaliste tchèque, qui fut à

8. Une formule reprise directement de Deleuze (Cinéma 2 - L'image-temps, Minuit, p. 105).

9. C'est du moins ainsi que l'interprète Alberto Gualandi (Deleuze, Paris, Les Belles Lettres, 1998), interprétation qu'endosse pleinement Cardinal. 
la fois son ami et son exécuteur testamentaire. S'autorisant de cette intimité, il a inscrit toute l'œuvre de Kafka sous la figure du père. Luttant contre cette réduction familialiste, un thème chez eux récurrent depuis L'anti-Edipe, Deleuze et Guattari soutiennent, dans leur ouvrage, ce qui n'est un paradoxe qu'en apparence: pour assurer une véritable lecture politique, il vaut parfois mieux ne pas trop connaître l'écrivain. S'interrogeant sur le bon usage d'un auteur, quel qu'il soit, Giroux abonde dans le même sens, et elle fait de cette méconnaissance une condition d'accès à l'œuvre, laquelle rend possible, chez «l'interprète-guerrier», une «justice herméneutique supérieure» (p. 178). On comprend évidemment que, pour interpréter selon ces lignes directrices, il faut distinguer soigneusement connaissance de l'œuvre et méconnaissance de l'individu, sans quoi un tel paradoxe (apparent) saperait ce Contr'hommage à la base...

Avec le texte suivant, "Pourquoi s'être rencontrés pour parler de Deleuze, pourquoi écrire maintenant sur son œuvre?»(p. 161-175), on passe de la politique de l'interprétation à son éthique. René Lemieux veut, en effet, élucider l'éthique deleuzienne de la lecture, en prenant appui sur son interprétation de Spinoza. Sylvano Santini, enfin, dans un texte intitulé "L'appropriation pragmatique de Deleuze aux États-Unis, ou en finir avec la nécessaire banalité de l'hommage " (p. 191-212), analyse la réception de l'œuvre deleuzienne chez notre voisin américain ${ }^{10}$. Pour ce faire, il fait appel à une comparaison assez osée, l'hommage médiéval, plus précisément le lien de vassalité, dont il prétend retrouver les trois étapes dans la réception américaine de l'œuvre de Deleuze: la condition de l'hommage, sa représentation cérémoniale et son symbole. Malgré quelques lueurs passagères, disons que ce cadre comparatif, assez artificiel, ne convainc guère.

Aidé du doctorant Francis Lapointe, Lawrence Olivier, connu pour ses travaux en philosophie politique, nous propose, pour clore cet ouvrage composite, une postface intitulée: "Gilles Deleuze philosophe: un nom propre» (p. 213-229). Nos deux auteurs veulent "poser la question de la pensée» (p. 216) et, pour ce faire, s'interrogent sur ce qui fait de Deleuze, l'individu, un philosophe. À cette fin, ils veulent ressusciter le sens même du geste philosophique. Évoquant d'abord une formule de Foucault, ils soutiennent que "l'invitation à philosopher [implique] quelque chose comme une pratique du gouvernement de soi ». Sur le plan méthodologique, cela suppose un lien critique à l'actualité (p. 218). Dans un esprit très hégélien, ils ajoutent que «c'est la tâche de la philosophie que de dire la vérité [d'une] époque, de la conduire vers son humanité ». Deleuze peut-il nous y aider? Oui, car il nous enseigne tout d'abord "que la réflexion philosophique doit se vivre, pas seulement s'apprendre par cœur»(p. 221). Il nous montre ensuite le chemin du nomadisme conceptuel, «une disposition à voyager sur de nouvelles scènes de la pensée » (p. 222). Ce qui implique que l'on accepte

10. C'était d'ailleurs le sujet de sa thèse de doctorat. 
le présent comme «l'éternel "point de fuite" du sens" et le concept comme ce qui " "dit l'événement, non l'essence ou la chose" " (p. 223; les auteurs soulignent). Or, comme l'événement, cet incorporel, dit Deleuze, est unique, le concept qui veut en capter le sens doit être constamment rejoué, sinon il ne pourra " "rendre justice" à la splendeur de sa singularité » (p. 225; les auteurs soulignent). Même alors, il ne faut jamais oublier que le concept, qui cherche à combler l'écart entre signe et sens, «n'est [...] qu'une possibilité composée à partir d'un champ infini d'effets de sens possibles» $(\text { p. 227 })^{11}$.

Avant de terminer, il faut dire un mot des illustrations accompagnant la couverture de l'ouvrage, ainsi que les divers textes. Elles ont été réalisées par Martin tom Dieck, un bédéiste professionnel d'origine allemande, professeur à la Folkwang-Hochschule (Essen), lequel avait rendu hommage à Deleuze dans deux albums antérieurs: Salut, Deleuze! (2000) et, en 2001, Les nouvelles aventures de l'incroyable Orphée (Le retour de Deleuze), tous deux produits en collaboration avec le journaliste et écrivain allemand Jens Balzer.

Au terme de notre petit périple, que retenir de ce contr'hommage et de ses trois niveaux superposés, "Offrande à un ami inconnu» (p. 14) ? La nature hétérogène de l'ouvrage et son caractère hybride sont-ils compensés par la solidité des analyses et par l'originalité de l'ensemble? Selon nous, oui, mais pas totalement. Côté analyse, on retiendra surtout les contributions rigoureuses et éclairantes de Beaulieu, Cardinal, Giroux et OlivierLapointe, ainsi que celle de Dantec, pour son entrelacs référentiel et la pertinence du témoignage. Il s'agit d'additions valables au corpus interprétatif deleuzien, qui n'a cessé de s'enrichir depuis sa mort, en 1995. Évidemment, nous n'avons offert ici qu'un aperçu sommaire des travaux de ce recueil, qui ne leur rend certainement pas justice. Car, bien que les diverses contributions soient d'inégale valeur, ce collectif atteint malgré tout son but: manifester la fécondité de l'œuvre deleuzienne et son rayonnement dans tous les champs de la culture. Que ces domaines soient aussi multiples que variés ne doit pas surprendre: au bout du compte, Deleuze avait érigé le nomadisme en principe, et il pratiquait allègrement l'ouverture de nombreuses lignes de fuite. Aussi ne serait-ce sans doute pas trahir les trois sections de l'ouvrage que de les qualifier d'interprétations (I), de réceptions (III) et de disséminations (II), bref, d'y décrypter les divers niveaux du travail de l'œuvre. En ce sens, ce recueil est assez unique, et peut-être fallait-il le Québec pour le produire, cet État-charnière, ce pays en perpétuelle gestation, où se joignent génie théorique continental et pragmatisme anglo-saxon. Le résultat est multiple et un rien éclaté ? Certes, mais il est assorti au pro-

11. Notons en terminant le survol de ce texte, à la fois tonique et éloquent, volontairement naif au second degré, qu'il est vraiment dommage que de trop nombreuses coquilles le gâtent — cinq dans la seule page 226 ! Un travail éditorial plus soigné aurait dû faire disparaître ces scories agaçantes. 
gramme deleuzien, qui préconisait l'expérimentation ouverte et récusait tout monopole du sens. Après tout, Dionysos a bien été démembré, et la dispersion contrôlée a aussi son charme.

JEAN-CLAUDE SIMARD

Collège de Rimouski

\section{Euvres de Charles De Koninck, tome I, Philosophie de la nature et des sciences, vol. 1, Québec, PUL, 2009, 475 P.}

Cette publication rend disponible une œuvre majeure de l'histoire de la pensée québécoise au $\mathrm{xx}^{\mathrm{e}}$ siècle. En effet, en plus d'être le père de plusieurs chercheurs universitaires reconnus et d'avoir laissé son nom à un pavillon de l'Université Laval, Charles De Koninck fut un animateur important de la vie philosophique et intellectuelle du Québec. Les PUL comptent publier ses œuvres complètes, dont voici l'ouvrage inaugural.

On a ordonné les textes selon une logique thématique, et c'était justice d'ouvrir le tout par la philosophie des sciences et de la nature, champ de prédilection de l'auteur. Rappelons-le, c'est dans ce domaine qu'il enseigna; d'ailleurs, sa thèse de doctorat en philosophie (Louvain, 1934) portait sur les travaux de l'astrophysicien Eddington.

Ce volume regroupe neuf textes, échelonnés de 1936 à 1960. On y trouve d'abord deux ouvrages: Le cosmos (1936) et L'univers creux (1960, inédit en français), ainsi que l' 'Introduction à l'étude de l'âme» de 1947, l'importante préface au Précis de psychologie thomiste de l'abbé Stanislas Cantin. On y a ajouté six articles: "Le problème de l'indéterminisme» (1937), «Mathématiques et philosophie» (1940), "Les sciences expérimentales sont-elles distinctes de la philosophie de la nature ?» (1947), «Un paradoxe du devenir par contradiction" (1954), ainsi que "Le problème de l'évolution" et "La responsabilité morale du scientifique" (inédit), tous deux de 1950. L'ensemble est précédé d'un avant-propos de Thomas De Koninck, ainsi que d'une présentation d'Yves Larochelle, maître d'œuvre de ce premier volume, lequel a aussi traduit les deux textes anglais. Un index onomastique et une bibliographie des œuvres du philosophe consacrées aux sciences viennent clore le tout.

Moins étudié sans doute que Maritain et Gilson, De Koninck constitue pourtant un relais cardinal de la première moitié du dernier siècle. Évidemment, la facture de ces textes témoigne d'une époque révolue; l'auteur y conjugue en effet trois niveaux distincts: les développements scientifiques, l'hylémorphisme aristotélicien et la théologie catholique. Comme le dit Larochelle dans sa présentation, ce style trouve encore des adeptes à l'Uni-

* Je remercie l'évaluateur anonyme de Philosophiques pour ses commentaires sur la version initiale de cette recension. 